\title{
1) Chemical Nature of IgM
}

\author{
Kaoru ONOUE \\ Department of Biochemistry, Kyushu University School of Dentistry
}

The paraproteins produced in patients with myeloma have been identified to be the same class of proteins as the immunoglobulins present in normal serum. Similarly chemical and antigenic properties of the macroglobulins produced in Waldenström's macroglobulinemia are similar in many respects to $\operatorname{Ig} M$ in normal serum or induced antibodies of IgM class. It is particularly interesting that some of the myeloma proteins have combining sites on defined parts of the molecules like antibodies. With Waldenström IgM proteins, Metzger's group found several such proteins, among which, one had combining activity to $\mathrm{Fc}$ portion of $\operatorname{IgG}$ and the other to nitrophenyl group. In addition, the chemical and functional uniformity of such proteins suggests the monoclonal nature of these proteins. These findings seem to support the attractive concept that each of these proteins is produced by neoplastic cells proliferated from a single clone which otherwise might produce antibody in normal process, and hence, each myeloma protein might represent each antibody. Thus, the comparison of these representatives with the antibodies normally induced by antigen stimulation is very important.

We report and discuss the results of structural studies on Waldenström IgM protein in comparison with the results of the structure and antibody function of rabbit IgM antibodies.

The studies on Waldenström IgM by Miller and Metzger $(1965,1966)$ have shown that the $\operatorname{IgM}$ (MW 900,000) molecule is composed of 5 subunits (MW 180,000) being linked through single disulfide bond each other to form probably a circular pentamer. Studying also a Waldenström IgM of the $L$ chain type of $\kappa$, we obtained additional results which support the above structure. Reduction of the purified $\operatorname{IgM}$ by $0.2 \mathrm{M}$. mercaptoethanol dissociated the molecule to $7 \mathrm{~S}$ subunits (IgMs). The IgMs produced was homogeneous in ultracentrifugal analysis and gel filtration and no release of free L chain as reported by Suzuki and Deutsch was observed. Upon gel filtration in $1 \mathrm{M}$. propionic acid the $\mu$ chain and $\kappa$ chain were obtained in the yields of $81 \%$ for $\mu$ chain and $19 \%$ for $\kappa$ chain. These results were consistent with 4 chain structure $(2 \mu$ and $2 \mathrm{~L})$ of the IgMs. Papain digestion of IgMs yielded two types of fragments. One of these fragments had an approximate molecular weight of 50,000 and was shown to be Fab $\mu$ fragment. This was confirmed by isolation and antigenic analysis of the component chains ( $\kappa$ chain and Fd $\mu$ chain) and comparison of the amino acid compositions of the fragment and its component chains. The yield 
of the Fab $\mu$ was consistent with the presence of $10 \mathrm{Fab} \mu$ per molecule of IgM. The other fragment was $3 \mathrm{~S}$ and identified as $\mathrm{Fc} \mu$. Thus the Fc fragment of the IgM was actually isolated. In contrast to $\operatorname{Ig} G$, the $F c$ portion of $\operatorname{Ig} M$ was more sensitive to papain digestion.

Pepsin digestion of the intact IgM yielded three types of fragments, $\mathrm{F}\left(\mathrm{ab}^{\prime}\right)_{2} \mu$, Fab $\mu$ and Fab piece. The $\mathrm{F}\left(\mathrm{ab}^{\prime}\right)_{2} \mu$ was a dimer and dissociated to its monomer form $\mathrm{Fab}^{\prime} \mu$ by reduction and alkylation. This monomer $\mathrm{Fab}^{\prime} \mu$ was larger and carried some additional antigenic determinant than Fab $\mu$ indicating the presence of $\mu$ chain determinant on the hinge region linking two $\mathrm{Fab}^{\prime} \mu$. This determinant was distinct from Fc $\mu$ determinants. The Fab $\mu$ piece was an interesting fragment in that it was a part of Fab and lacked some parts of both $\mathrm{Fd} \mu$ and $\kappa$ chains. Some results were obtained to suggest that the part lacked in Fab $\mu$ piece is probably C-terminal region.

When the intact IgM was digested with activated papain in the absence of reducing reagent, a high molecular weight $\mathrm{F} c \mu$ fragment was obtained in addition to Fab $\mu$. This $10.6 \mathrm{~S} \mathrm{FC} \mu$ fragment had a molecular weight of 320,000 and dissociated into $3.2 \mathrm{~S} \mathrm{FC} \mu$ units by reduction and alkylation. Thus the $10.6 \mathrm{~S} F \mathrm{c} \mu$ was considered to be a pentamer of $\mathrm{Fc} \mu$ or $\mathrm{F}(\mathrm{c})_{5} \mu$. The actual isolation of this fragment confirmed that disulfide bonds which link five IgMs are located on Fc portion of the IgM molecule. Thus our structural studies support the pentamer form of IgM in which each subunit (IgMs) is linked by disulfide bonds located on Fc region and four chain structure of IgMs. Supporting results were obtained by recent electronmicroscopic studies of $\operatorname{IgM}$ which showed a circular pentamer of IgMs joined in a central ring formed with Fc region of each IgMs (Svehag and collaborators 1968, 1969).

Interesting results were also obtained on the antibody combining sites of IgM. With rabbit anti-hapten antibodies, we first detected 5 combining sites per molecule. This number was only half of that predicted from the presence of 10 Fab fragments per molecule. In later works, Merler et al. (1968) and Onoue et al. (1968) were able to demonstrate 10 combining sites per molecule. However, in latter case, the affinity of about half of the combining sites were much weaker than the other half. From these results, we suggested the possible presence of 5 high affinity and 5 low affinity sites on a some molecule. This was to explain the facts that nearly all the previous investigations including our own have found only 5 combining sites. Recently, however, Ashman and Metzger found a Waldenström $\operatorname{IgM}$ which had combining activity to nitrophenyl group. They showed that the intact molecule as well as IgMs, $\mathrm{F}\left(\mathrm{ab}^{\prime}\right)_{2} \mu$ and $\mathrm{Fab} \mu$ all bind 1 mole of hapten per mole of heavy-light chain pair in each molecular species with uniform affinity. From these results they suggested that the results obtained in other works might well be explained by heterogeneity in antibody population.

Attempts were made if one can fractionate the antibody populations into the groups, one with relatively high affinity sites and the other with low affinity. In 
the first experiments, adsorption of anti-NS (azonapthalenesulfonate) antibodies on specific immunoadsorbent was inhibited by hapten. Similar inhibition curves were obtained for IgG, $\mathrm{F}\left(\mathrm{ab}^{\prime}\right)_{2} \gamma$ and IgM. However, the adsorption of Fabr was inhibited more effectively. This tendency was similar for Fabpep $\mu$ excepting half of this fragment was unable to bind with adsorbent in the absence of hapten. The curve for $F\left(a b^{\prime}\right)_{2} \mu$ was between the two curves. No tendency was shown in this type of experiments that the $\operatorname{IgM}$ antibodies or $\mathrm{F}\left(\mathrm{ab}^{\prime}\right)_{2} \mu$ could be fractionated. The different behaviour of the $\mathrm{F}\left(\mathrm{ab}^{\prime}\right)_{2} \mu$ might suggest the high and low affinity sites on a single $\mathrm{F}\left(\mathrm{ab}^{\prime}\right)_{2} \mu$ molecule. In the second experiments, purified IgM antibody was adsorbed by a limited amount of immunoadsorbent so that only $50 \%$ of the total antibody was adsorbed. The adsorbed antibody was eluted with hapten. The hapten binding properties of these two fractions of antibodies were examined by equilibrium dialysis. Both showed the presence of two sets of sites, about $50 \%$ of high affinity sites and the other $50 \%$ of low affinity. There was little tendency to show that the $\operatorname{IgM}$ antibodies were fractionated into the two groups of antibodies, one predominantly with ten high affinity sites and the other with ten low affinity sites. The results seemed rather favor for the presence of the two sets of sites on a single molecule. Thus we feel that our proposed possibility still remains valid for further test.

Most of the present work has been performed in collaboration with Dr. Tadamitsu Kishimoto of the same Department. 